Smith, Raymond, Wood, Julia, Jones, Fiona, Turner, Sue and Hurley, Michael (2019) A qualitative study exploring therapists' experiences of implementing a complex intervention promoting meaningful activity for residents in care homes. Clinical Rehabilitation, 33(3), pp. 575-583. Copyright (C) 2018 (The Authors). DOI: https://doi.org/10.1177/0269215518815233. 


\title{
A qualitative study exploring therapists experiences of implementing a complex intervention promoting meaningful activity for residents in care homes
}

\author{
Abstract \\ Objective: To explore the experiences of occupational therapists and physiotherapists and \\ to reveal any factors that can facilitate delivering a complex care home intervention \\ promoting meaningful activity.
}

Design: Qualitative interview study using data from three focus groups conducted longitudinally post intervention implementation. Data were analysed thematically.

Setting: Three residential care homes in South London, United Kingdom.

Subjects: All therapists involved in the implementation of the intervention: three occupational therapists and three physiotherapists.

Results: Three interconnected themes emerged from the analysis: (1) developing trusting relationships; (2) empowering staff; and (3) remaining flexible. Therapists described how successfully implementing a complex care home intervention was dependant on developing trusting relationships with care staff. This enabled the therapists to empower care staff to take ownership of the intervention and help to embed it in care home culture, facilitating long-term change. The therapists described how remaining flexible in their approach helped keep care staff engaged for the duration of implementation.

Conclusions: This study has revealed several important factors that can help facilitate therapists delivering complex interventions in care homes.

Key words: complex intervention; implementation; occupational therapy; experiences; care homes 


\section{Introduction}

The experiences of therapists delivering complex rehabilitation interventions to residents in care homes is poorly researched, with the various challenges in implementing programs within unpredictable environments a potential factor. As the first step towards a proposed research study, a complex intervention was implemented, entitled Active Residents in Care Homes (ARCH). This paper reports the experiences of the occupational therapists and physiotherapist throughout implementation delivery.

Various educational and training interventions have attempted to improve care staff practice in care homes with mixed success, with challenges such as high staff turnover and workload being barriers to effective implementation ${ }^{1}$. Sustainability of care home interventions is a well-documented challenge, with limited quantitative evidence that care home interventions are sustained soon after implementation, and no evidence they are maintained for longer than six months ${ }^{2}$. However, qualitative evidence suggests contextual factors unique to each care home (e.g. staff morale and interpersonal relationships with other staff and residents) are important factors to consider for optimising delivery of care home interventions and facilitating long-term change ${ }^{3}$.

Care staff (also known as paid caregivers, healthcare assistants and care workers) support people with various mental and physical health conditions in residential and nursing care facilities throughout the world. Research has consistently reported them as feeling under pressure with competing job demands, such as several care home residents needing assistance at once ${ }^{4}$. Evidence has shown that care staff who perceive they have had inadequate training to cope with the potential challenges of working with people with dementia feel under more strain and unconfident in their caring abilities ${ }^{5}$. More recently, research has shown care staff who have received appropriate training are more likely to deliver person-centred care and have higher job satisfaction ${ }^{6,7}$. Training for care home staff who work with people with dementia has been shown to improve knowledge, but this new knowledge is not always maintained long-term ${ }^{8}$. Further, training delivered as part of care home interventions is not always effective and often little consideration is given to how training will contribute to long-term intervention sustainability ${ }^{9}$. 
Evidence suggests providing generic training is unlikely to be enough to facilitate positive change in care staff practice and improve health outcomes for care home residents. Interventions which emphasise collaboration through staff working together whilst receiving clinical support are often reported as most beneficial ${ }^{10}$. Interventions that provide collaborative, face-to-face practice based training, which are delivered by experienced clinical facilitators, have been shown to be most effective at promoting positive change in care settings ${ }^{11,12}$. Further, the chances of successful implementation and maintenance of interventions are increased if support is continued after completion, and with the continued engagement of staff and families ${ }^{13}$.

The limited available evidence for successfully implementing care home interventions and promoting positive change is not only conflicting, little attention has been given to the experiences of those delivering the interventions. Therefore, the aim of this qualitative study was to explore the experiences of therapists and reveal any factors which can facilitate in delivering a complex intervention in three UK care homes.

\section{Methods}

This qualitative research formed part of the larger $\mathrm{ARCH}$ feasibility study ${ }^{14,15}$. The participating care homes catered for older people (aged over 65 years) with or without a diagnosis of dementia. Ethical approval was gained from the National Research Ethics Service (NRES) Committee London - South East in September 2014 (ref 14/LO/1329). The trial is registered as ISRCTN24000891.

A longitudinal qualitative design was employed in order to explore in-depth the experiences of therapists delivering the $\mathrm{ARCH}$ intervention during different phases of implementation. Focus group interviews were utilised and were conducted alongside intervention delivery.

\section{The Active Residents in Care Homes (ARCH) intervention}

$\mathrm{ARCH}$ is an occupational therapy led intervention which utilises a holistic whole systems approach (for example: considers individual resident needs, staff skills, organisational processes and care home environments) to increase meaningful activities and improving the wellbeing of care home residents. Occupational therapists were supported by 
physiotherapists and therapy assistants in intervention design and delivery. ARCH aimed to improve the health and quality of life of residents in care homes through increasing their level of participation in meaningful activity and to increase the confidence and skills of care staff. ARCH comprises of two phases lasting one year in each care home:

Implementation phase (0-4 months): The therapists work in collaboration with care staff in each care home to integrate the $\mathrm{ARCH}$ programme into everyday working practices. At the start of this phase the therapists spend time getting to know the care staff, residents and the unique working environments of each care home. It is during this phase that care staff undertake ten training modules designed specifically for the $\mathrm{ARCH}$ programme and tailored to the requirements of each care home (Table 1). The training encourages staff to think about residents' individual needs, through the teaching of theory behind behaviour perceived as challenging, and ways in which they can improve their working practice. Alongside formal training, care staff receive one-to-one on the floor support and rolemodelling to help improve their caring confidence and are assigned a resident who they work with closely to implement their learning.

Consolidation phase (5-12 months): The therapists complete a staged withdrawal from the home leaving the rehabilitation assistant to support managers, staff, residents and families to carry on supporting residents with activities. Staff are supported to take responsibility for the programme to enable sustainability once the rehabilitation assistant leaves at month 12 .

\section{Participants and setting}

All occupational therapists and physiotherapists involved in the delivery of ARCH were verbally asked if they would like to take part in focus groups. It was explained they were under no obligation to participate, could withdraw at any time and that all responses would be anonymised. All therapists who were asked if they would like to take part did so. Written consent was gained from all participants prior to interview and it was verbally repeated that they do not have to share any information with the group with which they were uncomfortable. Care was taken to ensure participants felt relaxed in their surrounding and that confidentiality could be maintained during interviews. 


\section{Data collection}

Data were collected using focus group interviews ${ }^{16}$ by RS, SK and JW; all researchers had experience in conducting in-depth interviews and focus groups with health professionals. The approach was exploratory and focus groups were chosen as a data collection method to enable the collection of wide ranging insights in a single setting ${ }^{17}$. Focus groups also enabled participants to agree or disagree with what was said, refine their own thoughts and opinions based on what others reported, and share experiences with each other that would not have been possible in one-to-one interviews ${ }^{18}$.

Topic guides were used for all three focus groups to encourage participants to discuss their experiences. Topics included, for example, skills needed for successful implementation, challenges encountered and how they were overcome, and how they formed and maintained relationships with care staff and residents. The focus groups were semistructured and participants were given time to discuss additional topics they felt were not covered during the interviews.

The focus groups were conducted at three time points: just after implementation (time 1); after one year (time 2); and two years later (time 3 ) at three UK care homes. All six therapists involved with intervention delivery, three occupational therapists and three physiotherapists, took part. A minimum of three and maximum of four therapists were present during each focus group. Focus groups lasted 123, 57 and 40 minutes respectively, and were held in private rooms at either one of the participating care homes or at the therapists' place of work. All participating therapists were female.

\section{Data analysis}

Qualitative data gathered from the focus groups were transcribed, anonymised and analysed ${ }^{19}$ using thematic analysis ${ }^{20}$. Thematic analysis is a rigorous and commonly utilised approach to generate themes from qualitative data ${ }^{21}$. Initially, all transcripts were read by one researcher (RS) to allow familiarisation with the data and make notes about the emerging themes from each. A second researcher (JW) then read the transcripts independently and made notes about themes and how these relate to those identified by RS. Through re-reading transcripts, discussion and refinement, both researchers agreed about the emerging themes. 
To ensure trustworthiness of the findings, member checking ${ }^{22}$ was completed by sending the emerging themes to the therapists, providing them with the opportunity to comment on the analysis prior to final refinement of the findings. The findings were reported using the Consolidated Criteria for Reporting Qualitative Research (COREQ) checklist, a comprehensive 32 item checklist to ensure rigor in studies involving interviews or focus groups ${ }^{23}$.

\section{Results}

Three overarching interconnected themes were identified: 1) developing trusting relationships; 2) empowering staff; and 3) remaining flexible. Anonymised quotes from participants are presented to illustrate the themes.

\section{Theme 1: Developing trusting relationships}

Building trusting relationships with the care staff was described as crucial to the success of implementing $\mathrm{ARCH}$. Therapists talked about how they needed adequate time for preintervention work in order to understand the individual workings of the care homes and for the staff to get to know them and dispel any concerns they were being assessed or scrutinised. Although this added time to the intervention, therapists described it as an important part of the process and it helped them to build trusting relationships with care staff:

...as we've realised that there's more information that needs to be given to care homes before going in. So setting that sort of foundation of continuing to give the same message over and over and it's not just once off, so that foundation work...has made it a lot easier. As well as...in terms of the skill building relationships with people, building trust and knowing that, showing them that we're on their side and we're not some kind of assessing body." (Therapist A: time 2).

The importance of building and maintaining trust throughout the training and $\mathrm{ARCH}$ delivery was also described as important. The therapists reported that this was achieved through thoughtful use of language when talking to care staff so that it was positive and encouraging. For example, a therapist explained how considerable effort was made not to be viewed as overly critical of the care staff and their working practice: 
"I think that's why even giving the macro assessment feedback had to be so painstakingly worded that it wasn't implicating anybody, we weren't pointing fingers, we were trying to positively construct it" (Therapist A: time 1)

Therapists talked about requiring certain skills and abilities in order to successfully build trusting relationships with care staff. Whilst the ability to work as part of a multidisciplinary team, good team working and communication skills were all perceived as important, emotional intelligence and 'people skills' were described as imperative for therapists to have. A senior occupational therapist discussed the personal characteristics she looked for when recruiting for $\mathrm{ARCH}$ :

“...ideally you'd want someone...that has some kind of clinical background in what we're trying to achieve first of all, so in dementia care, in working with older people. I'd look for somebody who is confident to train staff but therefore you also need to be confident to know what the content is but it's not just about that, it is more about softer skills as well so getting a feel for that person, knowing how they treat others, how could they build relationships." (Therapist A: time 2).

Developing trusting relationships with managers and helping them to be actively engaged with $\mathrm{ARCH}$ was also described as an essential part of successful implementation. For example, therapists described how those managers who were engaged were more likely to give care staff time for or remind them to attend the training. When asked how easy or difficult it was to get care staff to attend ARCH training, a therapist replied:

"I mean we didn't get bad attendance rates, it was quite good and we certainly had the deputy manager on our side...She (deputy manager) was a good force of making sure that people (care staff) got there." (Therapist B: time 1)

Maintaining boundaries and remaining impartial when it came to disagreements between staff members or between care staff and management was also challenging but necessary so as not to damage the trust they had developed. The therapists were able to do this by focussing on what they were there for, using their communication skills to try and deescalate negative situations and not take sides when disagreements occurred: 
"...we also are really having to maintain a neutral ground always between

relationships. We can't take sides, we can't be seen to just be on the manager's side against the... So that is, it's really hard to kind of show empathy and also to show understanding but also maintain neutrality...I think there's a few situations sometimes that arise where you have to be quite skilled in knowing, and not pitching people against each other..." (Therapist A: time 1)

Along with developing trusting relationships, therapists also talked about ways in which they were able to empower staff.

\section{Theme 2: Empowering staff}

Empowering care staff was an important topic discussed by the therapists. They worked collaboratively with care staff to encourage and empower them (e.g. increased sense of authority and control) to take ownership of ARCH which was seen as the best way to embed the intervention in care home culture for the long-term. In particular, they talked about their perceptions of the impact of $\mathrm{ARCH}$, with some describing how using a solution focussed approach had enabled care staff to think for themselves and problem solve. This was most often seen around how best to deal with challenges and engage in meaningful activities with residents:

"So sometimes helping them to access not giving them the solution but saying okay, so I hear that this is hard and this is hard, you know, do you feel like you, this can change in any way or is there anything you could do to try and communicate this to somebody?." (Therapist D: time 3)

Acknowledging staff difficulties and reminding them that they were working in collaboration, as opposed to instructing them, helped to keep care staff engaged with the intervention and also enabled the therapists to continue empowering them:

"One of them, she's great, very passionate about what she does but was avoiding a one-to-one interview so you acknowledge that and then went through her manager who allowed it and so that gave her that freedom to then have an interview of what she had seen as really important...Acknowledging the difficulties and the culture of the care home as well". (Therapist D: time 3) 
The therapists discussed witnessing positive changes in care staff abilities and attitudes throughout implementation. For example, they perceived staff felt their jobs were more worthwhile, enjoyable, were more confident in their abilities and that this had led to an increase in morale. These mood and confidence changes ultimately led the therapists to notice that care staff behaviours were changing as they were becoming more involved with the residents, in particular by initiating group activities or spending time talking to residents on an individual basis:

"I think the changes we saw, one of the biggest ones was that staff was more engaged with the residents I would say. So instead of just like walking past and sometimes they'd walk past and they'd still say hello to them but they'd get them engaged in doing stuff, they were more willing to do activities without us always being the ones to instigate it" (Therapist B: time 1)

Formal training sessions and on the floor role modelling were important aspects in empowering care staff, with the role modelling embedding learning from the formal training sessions. Therapists' working on the floor was also viewed as offering staff the opportunity to be actively engaged in implementing positive change from the $\mathrm{ARCH}$ training, something viewed as important for sustainability positive changes in staff behaviour:

"I think that became the learning throughout that actually people [residents] can do way more than we think but also that we have to bridge the gap between allowing them to do, giving them opportunities to do that by filling those gaps of the impairments that they have...So I think just both from a resident perspective in that but also the staff sort of seeing and learning oh wow, we can actually give people more to do and they can do more than we think." (Therapist A: time 1)

Building trusting relationships and empowering care staff were described by therapists as important aspects to the successful implementation of the ARCH intervention. However, without remaining flexible in their approach to delivery and the workings of each individual care home, this may not have been possible. 


\section{Theme 3: Remaining flexible}

The therapists explained how remaining flexible in their approach to delivering $\mathrm{ARCH}$ and training modules was important throughout implementation. Without a degree of flexibility, the development of trusting relationships and empowering care staff may not have been possible. In particular, understanding that each care home was unique, operated differently, had staff with various levels of prior training and needed context specific training was part of the therapists learning process. For example, after implementing $\mathrm{ARCH}$ in the first care home the therapists learned that, whist it was important to have a structure for implementation to refer back to, it was not possible to rigidly stick to it:

"...you almost have this background skeleton of structure to ground you but then within that you can be flexible because you have to be in this kind of environment, you can't just stick to structures." (Therapist A: time 2)

Some care staff discussed with the therapists that they felt unable to complete the ARCH related training tasks and were reluctant to come to some training modules, with lack of time and pressure to complete other work responsibilities taking priority. In order to increase attendance, the therapists adopted a friendly and flexible approach, which was non-blaming, to overcome reported care staff challenges:

"...some people felt quite under pressure to do the tasks that we were setting them. I remember some people saying I'm not going to come to the next session because I haven't managed to do it, you know, I haven't done the work and I don't want to, I'm not going to come to a training. And it was...having a friendly approach really and just saying look, it's more important that you attend the training, don't worry if you haven't had time, you know, trying to work out ways around the problems that people were having." (Therapist E: time 1)

This same flexibility around enabling care staff to attend training continued into the sessions themselves. In particular, the therapists discussed being mindful of being pragmatic and tailoring the training to the challenges and unique environments of each care home in order to try and increase engagement. As reported by one therapist, assessing care staff engagement and subsequently modifying the types of training delivery during each session was essential to maintain staff engagement: 
"I think gauging how invested they were in the sessions. So if we could gauge they were zoning out we could introduce different methods whether it's getting them participating, doing questions, role plays, and so sort of assessing where they're at and actually when they were overloaded with information knowing to stop or what to, what were the priority points, key points to get the message across." (Therapist D: time 3)

The therapists maintained a degree of flexibility throughout implementation, however they talked about becoming progressively inflexible surrounding the priority of $A R C H$ training to encourage staff attendance. When beginning $\mathrm{ARCH}$ the therapists described being overly concerned with being too assertive, but they suggested this had led some care staff to feel ARCH training was not important:

“...there are times when I think previously I hadn't been as assertive because I thought oh, you know, we don't want to step on toes duh de duh, but actually that's not helpful because then they learn that oh, it's not that important or I don't really have to be on time or if something else comes up, it's fine, the ARCH team can just come second". (Therapist A: time 2)

\section{Discussion}

The purpose of this study was to explore the experiences of therapists in delivering a complex care home intervention. The results showed that developing trusting relationships with care staff enabled the therapists to empower them to take ownership of the intervention, supporting long-term change. This was made possible by remaining flexible in their approach and helped keep care staff engaged for the duration of implementation.

However, there were challenges, specifically with the therapists initially being too flexible in their approach and finding it difficult to engage some care staff to prioritise ARCH training due to pressures of completing care related tasks. This has been found previously, with care staff reportedly often feeling under work-related pressure ${ }^{4,5}$. Through taking a collaborative approach and ensuring care staff were aware they were working in partnership, the therapists felt they were able to overcome many of the challenges encountered and care staff felt able to communicate their concerns to them. 
Retaining new knowledge and practice skills for staff working with people living with dementia is often lost over time ${ }^{8}$. However, the ARCH therapists felt that the tailored onthe-floor practice based learning helped to embed care staff knowledge from the formal training sessions and empower staff to sustain positive behaviour changes. This has been found by others, with face-to-face practice based learning, which has been developed specifically for staff relevant to their role and experience, shown to be most effective at effecting positive changes ${ }^{11}$.

Another significant aspect of tailored training combined with on the floor role modelling was that it was perceived by the therapists to increase care staff confidence in their caring ability, which ultimately led them to become more involved with residents and improve their care practice. Therefore, training needs to be flexible, context specific, take into account previous training care staff may have had and to explore reasons why some care staff are more engaged than others. This is an important finding, as previous research has shown better trained care staff are more likely to deliver person-centred care, have higher job satisfaction and offer improved quality of life for people living in care homes ${ }^{6,7}$.

The findings of this study should be considered with respect to its limitations. Firstly, this was a small sample qualitative study investigating the experiences of therapists implementing one intervention in three UK care homes. Further, each focus group consisted of either three or four participants, which is under the recommended six to twelve participants needed to yield sufficient diversity in collected data ${ }^{24}$. Therefore, it cannot be assumed the findings are applicable to therapists delivering intervention in different settings. Secondly, focus groups were chosen due to the rich data that would likely be produced from the therapists openly sharing their experiences with each other, thus generating new ideas and deeper understanding than individual interviews might alone ${ }^{17}$. Nonetheless, the focus group method has its limitations. For example, one dominant member taking over discussion, that they tend not to elicit emotional information and participants may over exaggerate or make up answers in order to hide a lack of knowledge ${ }^{25}$. However, in this study these potential weaknesses were mitigated by good facilitation by experienced interviewers. 
Delivering a holistic, activity based care home intervention using a whole systems approach can be challenging for therapists. Without the therapists taking adequate time before and during delivering $\mathrm{ARCH}$ to build trusting relationships with care staff, successfully engaging with care staff and facilitating positive behaviour change could have been compromised. Future care home interventions which require collaborative working between therapists and care staff, should consider facilitating additional time prior to starting implementation to allow for relationship building and trust to develop.

Further research is necessary to explore the experiences of the care staff themselves and how similar they are, or not, to those of the therapists. These multiple perspectives may enable deeper understanding of the challenges for all involved with care home research. It may allow for comparisons surrounding how interventions, such as $\mathrm{ARCH}$, become integrated into the care home culture and are sustained beyond the life of the project. Additionally, exploring the experiences of other clinicians and allied health professional involved with care home interventions would help in understanding the techniques they employ to overcome the various challenges they encounter.

\section{Clinical Messages}

- Care homes are challenging environments to deliver complex interventions, however this study highlights several factors that can maximise the probability of success.

- Therapists should consider the importance of developing and maintaining trusting relationships with care staff. Trusting relationships can enable therapists to empower care staff to take ownership of interventions, supporting long-term change.

- Remaining flexible in approach to intervention delivery can help keep care staff engaged throughout implementation. 


\section{References}

1. Low LF, Fletcher J, Goodenough B, Jeon H-Y, Etherton-Beer C, MacAndrew M, Beattie E. (2015) A systematic review of interventions to change staff care practices in order to improve resident outcomes in nursing homes. PloS One, 10(11), e0140711.

2. Rapaport P, Livingston G, Murray J, Mulla A, Cooper C. (2017) Systematic review of the effective components of psychosocial interventions delivered by care home staff to people with dementia. BMJ Open, 7(2), e014177.

3. Lawrence V, Fossey J, Ballard C, Ferreira N, Murray J. (2016) Helping staff to implement psychosocial interventions in care homes: augmenting existing practices and meeting needs for support. International Journal of Geriatric Psychiatry, 31(3), 284-93.

4. Vandrevala T, Samsi K, Rose C, Adenrele C, Barnes C, Manthorpe J. (2017) Perceived needs for support among care home staff providing end of life care for people with dementia: a qualitative study. International Journal of Geriatric Psychiatry, 32(2), 155-163

5. Morgan DG, Stewart NJ, D'Arcy C, Forbes D, Lawson J (2005) Work stress and physical assault of nursing aides in rural nursing homes with and without dementia special care units. Journal of Psychiatric and Mental Health Nursing, 12(3), 347-58

6. Scerri A, Scerri C. (2017) Outcomes in knowledge, attitudes and confidence of nursing staff working in nursing and residential care homes following a dementia training programme. Aging \& Mental Health, [Epub ahead of print]. doi: 10.1080/13607863.2017.1399342.

7. Zimmerman S, Williams CS, Reed PS, Boustani M, Preisser JS, Heck E, Sloane PD. (2005) Attitudes, stress, and satisfaction of staff who care for residents with dementia. The Gerontologist, 1(1), 96-105.

8. Spector A, Revolta C, Orrell M. (2016) The impact of staff training on staff outcomes in dementia care: a systematic review. International Journal of Geriatric Psychiatry, 31(11), 1172-1187.

9. Boersma P, van Weert JC, Lakerveld J, Dröes RM. (2015) The art of successful implementation of psychosocial interventions in residential dementia care: a systematic review of the literature based on the RE-AIM framework. International Psychogeriatrics, 27(1), 19-35.

10. Goodman C, Dening T, Gordon AL, Davies SL, Meyer J, Martin FC, Gladman JRF, Bowman C, Victor C, Handley M, Gage H, Iliffe S, Zubair M. (2016) Effective health care for older people living and dying in care homes: a realist review. BMC Health Services Research, 16:269.

11. Surr CA, Gates C, Irving D, Oyebode J, Smith SJ, Parveen S, Drury M, Dennison A. (2017) Effective dementia education and training for the health and social care workforce: A systematic review of the literature. Review of Educational Research, 87(5), 966-1002. 
12. Hall AJ, Watkins R, Lang IA, Endacott R, Goodwin VA. (2017) The experiences of physiotherapists treating people with dementia who fracture their hip. $B M C$ Geriatrics, 17:91.

13. Lawrence V, Fossey J, Ballard C, Moniz-Cook E, Murray J. (2012) Improving quality of life for people with dementia in care homes: making psychosocial interventions work. International Journal of Geriatric Psychiatry, 31(11), 1172-1187.

14. Koskela SA, Jones F, Clarke N, Anderson L, Kennedy B, Grant R, Gage H, Hurley MV. (2015). Active Residents in Care Homes (ARCH): study protocol to investigate the implementation and outcomes of a whole-systems activity programme in residential care homes for older people. Physiotherapy, [Epub ahead of print], doi:10.1016/j.physio.2015.12.001.

15. Smith R, Wood J, Jones F, Anderson L, Hurley M. (2017) Active Residents in Care Homes (ARCH): a holistic approach to promoting and encouraging meaningful activity for residents living in care homes (innovative practice). Dementia, [Epub ahead of print], doi:10.1177/1471301217727129.

16. McLafferty I. (2004) Focus group interviews as a data collecting strategy. Journal of Advanced Nursing, 48(2), 187-94.

17. Breen RL. (2006) A practical guide to focus-group research. Journal of Geography in Higher Education, 30(3), 463-475.

18. Hennink MM. (2014) Focus Group Discussions: Understanding Qualitative Research. Oxford University Press: New York.

19. Auerbach C, Silverstein LB. (2003) Qualitative Data an Introduction to Coding and Analysis. New York University Press.

20. Braun V, Clarke V. (2006) Using thematic analysis in psychology. Qualitative Research in Psychology, 3(2), 77-101.

21. Vaismoradi M, Jones J, Turunen H, Snelgrove S. (2016). Theme development in qualitative content analysis and thematic analysis. Journal of Nursing Education and Practice, 6(5), 100-110.

22. Morse JM. (2015) Critical analysis of strategies for determining rigor in qualitative inquiry. Qualitative Health Research, 25(9), 1212-1222.

23. Tong A, Sainsbury P, Craig J. (2007) Consolidated criteria for reporting qualitative research (COREQ): a 32-item checklist for interviews and focus groups. International Journal for Quality in Health Care, 19(6), 349-357.

24. Onwuegbuzie AJ, Dickinson WB, Leech NL, Zoran AG. (2009) A qualitative framework for collecting and analyzing data in focus group research. International Journal of Qualitative Methods, 8(3), 1-21.

25. Kruger RA, Casey MA. (2015) Focus Groups: A Practical Guide for Applied Research. London: SAGE Publications. 
Table 1: ARCH training modules

1. Introduction to $\mathrm{ARCH}$ and the wellbeing wheel

2. Importance of knowing the person

3. Communication

4. Wellbeing, ill-being and behaviour

5. Meaningful activity

6. Facilitating group activities

7. The environment

8. Mobility, activity and positive risk taking

9. Falls and medication

10. Sleep and arousal 